\title{
Efficacy of Rehabilitative Experience Declines with Time after Focal Ischemic Brain Injury
}

\author{
Jeff Biernaskie, Garry Chernenko, and Dale Corbett \\ Division of Basic Medical Sciences, Faculty of Medicine, Memorial University of Newfoundland, St. John's Campus, Newfoundland, Canada A1B 3V6
}

To maximize the effectiveness of rehabilitative therapies after stroke, it is critical to determine when the brain is most responsive (i.e., plastic) to sensorimotor experience after injury and to focus such efforts within this period. Here, we compared the efficacy of 5 weeks of enriched rehabilitation (ER) initiated at 5 d (ER5), ER14, or ER30 after focal ischemia, as judged by functional outcome and neuromorphological change. ER5 provided marked improvement in skilled forelimb reaching ability and ladder-rung-and narrow-beam-walking tasks and attenuated the stroke-induced reliance on the unaffected forepaw for postural support. ER14 provided improvement to a somewhat lesser extent, whereas recovery was diminished after ER30 such that motor function did not differ from ischemic animals exposed to social housing.

To examine potential neural substrates of the improved function, we examined dendritic morphology in the undamaged motor cortex because our previous work (Biernaskie and Corbett, 2001) suggested that recovery was associated with enhanced dendritic growth in this region. ER5 increased the number of branches and complexity of layer $V$ neurons compared with both social housing and control animals. Dendritic arbor after ER14 (although increased) and ER30 did not differ from those exposed to social housing. These data suggest that the poststroke brain displays heightened sensitivity to rehabilitative experience early after the stroke but declines with time. These findings have important implications for rehabilitation of stroke patients, many of whom experience considerable delays before therapy is initiated.

Key words: stroke; ischemia; rehabilitation; recovery of function; dendritic morphology; motor cortex; striatum; forelimb

\section{Introduction}

Previous studies have demonstrated that induction of proteins is associated with endogenous neural repair within the first 2 weeks after an ischemic insult. For example, levels of the growthassociated protein-43 (Stroemer et al., 1995; Kawamata et al., 1996) and superior cervical ganglion-10 (McNeill et al., 1999) are transiently elevated in areas surrounding and contralateral to the infarct during the first few weeks after injury. Moreover, expression of growth-promoting factors such as nerve growth factor (Dahlqvist et al., 1999) and basic fibroblast growth factor (bFGF) (Speliotes et al., 1996) are altered in the weeks after ischemia. These processes may engender the injured brain with greater plasticity, and during this period, the brain may be optimally responsive to rehabilitative experience.

After clinical stroke, commencement of physical rehabilitation programs varies from days to several weeks after the insult. Considering the time course of these endogenous neuroreparative events, the efficacy of rehabilitative therapy may vary consid-

Received Aug. 17, 2003; revised Dec. 3, 2003; accepted Dec. 5, 2003.

This work was funded by the Canadian Institutes of Health Research (CIHR) to D.C. J.B. was supported by a CIHR doctoral studentship. We are very grateful to Aleksandra Szymanska, Shirley Granter-Button, and Andrea DarbyKing for helpful advice and technical assistance, and to Dr. John McLean for use of the Center for Image Analysis. We also thank Dr. John Evans for statistical advice.

Correspondence should be addressed to Dr. Dale Corbett, Basic Medical Sciences, Faculty of Medicine, Health Sciences Center, Memorial University of Newfoundland, St. John's Campus, Newfoundland, Canada A1B 3V6. E-mail: corbett@mun.ca.

DOI:10.1523/JNEUROSCI.3834-03.2004

Copyright $\odot 2004$ Society for Neuroscience $\quad$ 0270-6474/04/241245-10\$15.00/0 erably depending on the time of its initiation. Therefore, it is imperative to determine whether there is a period during which the poststroke brain is most sensitive to physical rehabilitation to maximize the functional gains from such therapy.

We demonstrated previously that enriched living conditions in concert with daily rehabilitative reach training of the impaired forelimb enhanced neuronal growth in the cortex contralateral to the damaged hemisphere, which was associated with improved forelimb function (Biernaskie and Corbett, 2001). In that study, enriched rehabilitation (ER) was initiated 2 weeks after ischemic injury. Here, we hypothesized that implementing rehabilitative treatment earlier after the stroke, during this period of endogenous repair and remodeling, would further enhance functional outcome. To characterize this potential "critical period," animals received either social housing only or enriched rehabilitative training initiated at $5 \mathrm{~d}$ (ER5), ER14, or ER30 after middle cerebral artery (MCA) occlusion. Animals were assessed on sensorimotor function before treatment and 2 and 5 weeks after the initiation of rehabilitative treatment. After 5 weeks of therapy, dendritic morphology was examined within the motor cortex of the intact hemisphere and dorsolateral striatum to determine potential differences in neuronal reorganization.

Our hypothesis was confirmed such that early initiation of ER (ER5) provided enhanced functional outcome relative to ischemic animals receiving delayed ER (ER30). Improved outcome was also associated with greater dendritic complexity of layer $\mathrm{V}$ neurons. These results suggest that the efficacy of rehabilitative 
therapy after stroke is influenced by the time of its commencement. Extended delays may require more intense or longer duration therapy to achieve the same functional gains.

\section{Materials and Methods \\ Subjects}

Subjects were male Sprague Dawley rats $(n=40)$ weighing between 280 and $350 \mathrm{gm}$ at the time of surgery. Animals were housed on a reverse 12 hr light/dark cycle. Behavioral testing was done during the dark phase. They were provided with food and water ad libitum except during behavioral training and testing periods. All animals were housed in standard Plexiglas cages (two to three animals per cage) before and after surgery except for those exposed to ER, which were transferred to large wiremesh (enrichment) cages (Biernaskie and Corbett, 2001), four to six animals per cage at 5,14 , or $30 \mathrm{~d}$ after surgery, respectively. All procedures were in accordance with guidelines set by the Canadian Council on Animal Care and the Memorial University Animal Care Committee.

\section{Surgical procedures}

Anesthesia was induced using halothane (3\% induction, $1 \%$ maintenance) in $30 \% \mathrm{O}_{2}$ and $70 \% \mathrm{~N}_{2} \mathrm{O}$. A midline incision was made, and a burr hole was drilled in the skull overlying the dorsolateral parietal cortex. The vasoconstrictive peptide endothelin-1 (ET-1; $120 \mathrm{pmol}$ in $3 \mu \mathrm{l}$ of $0.9 \%$ saline) was stereotaxically applied to the MCA (proximal to the striatal branches) to produce prolonged vasospasm and subsequent ischemic injury (Sharkey et al., 1993; Biernaskie and Corbett, 2001; Biernaskie et al., 2001). Stereotaxic coordinates were as follows: anteroposterior, +0.9 $\mathrm{mm}$; mediolateral, $-5.2 \mathrm{~mm}$; dorsoventral, $-8.7 \mathrm{~mm}$. The needle remained in place for $5 \mathrm{~min}$ to minimize backflow. Rectal temperature was monitored and maintained between 36.5 and $37.5^{\circ} \mathrm{C}$ using a heated water blanket for the duration of the surgery. Control animals included those that underwent sham surgery consisting of either a $3 \mu$ injection of saline $(n=4)$ adjacent to the MCA or those that received a failed injection of ET-1 $(n=4)$, resulting in ischemic injury restricted to the site of injection (piriform cortex). Previous studies (Ward et al., 1998) as well as our own (Biernaskie and Corbett, 2001; Biernaskie et al., 2001) have found that because of anatomical variation of arterial vasculature in individual animals and insufficient accuracy in the placement of the injection needle, the success rate using this model for MCA occlusion is $\sim 50-60 \%$. Therefore, to reduce the number of animals used for this study, those animals with failed MCA occlusion and an absence of significant behavioral impairment $(\geq 80 \%$ of training performance on staircase and single-pellet reaching on days 3 and 4 after ischemia) were included as controls.

\section{Treatment conditions}

After behavioral assessment on days 3 and 4 after stroke, animals with similar behavioral impairments were grouped and randomly assigned to one of four treatment conditions consisting of either social housing only or ER. Ischemic animals that did not receive rehabilitative therapy (social housing, $n=9$ ) were housed in standard Plexiglas cages (two to three animals per cage). Nonischemic control animals $(n=9)$ were also housed in these social-housing conditions. The start of social-housing treatment was considered to be $5 \mathrm{~d}$ after ischemia, and animals were killed $40 \mathrm{~d}$ later (the same time course as ER5 animals).

Enriched rehabilitation. To examine the efficacy of rehabilitation at different times, ER therapy was initiated at either $5(n=8), 14(n=7)$, or $30(n=7) \mathrm{d}$ after the stroke. Enrichment consisted of four to six animals being housed in large wire-mesh cages filled with a variety of objects intended to stimulate general (bimanual) limb use. Procedures for rehabilitative reach training have been described previously (Biernaskie and Corbett, 2001). Briefly, animals were exposed for $6 \mathrm{hr}$ per day ( $5 \mathrm{~d}$ per week) to a Plexiglas reaching apparatus filled with highly palatable mini M\&Ms (M\&M/Mars, Hackettstown, NJ) used to encourage (rather than force) coordinated use of the impaired forelimb. The design of the apparatus prevented retrieval of pellets with the "good" limb. Socially housed animals and operated controls did not receive ER but were fed the average daily intake $(\sim 10 \mathrm{gm} / \mathrm{d})$ of M\&Ms retrieved by animals during a rehabilitation session. Notably, this amount of chocolate did not appear to have a stimulant effect, because animals reached vigorously at the beginning of each rehabilitation session, but this was almost always followed by a period of inactivity or sleep.

All animals were exposed to their respective treatment conditions and then reassessed for behavioral impairment at 14 and $35 \mathrm{~d}$ after initiation of treatment (regardless of when the treatment was initiated after stroke). Animals were killed after completion of the behavioral testing ( $40 \mathrm{~d}$ after initiation of treatment, regardless of start time) to examine the effects of ischemia and the treatment condition on neuronal reorganization within the undamaged hemisphere.

\section{Behavioral assessment}

Animals were trained before ischemic injury and tested on each of the behavioral tests at days 3 and 4 after ischemia (before treatment) and again after 14 and $35 \mathrm{~d}$ of either enriched rehabilitation (regardless of start time) or social housing only. For the duration of behavioral training and test periods, animals were food restricted to $12 \mathrm{gm} / \mathrm{d}$. Body weights were recorded every second day and did not fall below $85 \%$ of initial weight.

Single-pellet reaching. This task has been modified from those described previously (Peterson and Devine, 1963; Whishaw et al., 1991). Forelimb reaching requires fine motor skill of both proximal and distal musculature as well as sensation to successfully retrieve food pellets. The single-pellet task examined both gross ability to retrieve pellets and reaching accuracy, which is more sensitive to subtle impairments and compensatory reaching strategies that may not be detected by other reaching tests (i.e., the staircase test, described below). Animals were trained for $18 \mathrm{~d}$ to reach through a 1.1-cm-wide vertical slot to obtain a food pellet situated in a well $2 \mathrm{~cm}$ from the front of a Plexiglas box on a shelf $3.5 \mathrm{~cm}$ high. The slot was positioned $1.4 \mathrm{~cm}$ from the left wall of the reaching box to discourage use of the right (unaffected) paw (see Fig. $2 A$ ). For each test session, animals reached for 25 mini M\&Ms (presented one at a time) and were filmed with a Sony (Tokyo, Japan) TRV17 digital video camera for later analysis. Reaches were only considered successful if the pellet was eaten, whereas failed attempts included all limb advances resulting in a missed, displaced, or dropped pellet. Reaching success was expressed as the number of successful retrievals (of 25 pellets presented). Reaching accuracy was determined using the following equation: (number of successful reaches/total number of reaching attempts) $\times 100$. It should be noted that this task differed from the rehabilitative reach training task. During ER, animals reached into a trough filled with M\&Ms situated below the impaired paw. In contrast, the single pellet test required rats to reach to an elevated shelf directly in front of them for M\&Ms presented one at a time. One animal did not learn the single-pellet reaching test and so was excluded from statistical analyses.

Staircase skilled reaching. In addition to the single-pellet reaching test, the staircase reaching task was also used. This was done for a number of reasons: First, to assess the animals' ability to retrieve smaller pellets (relative to the mini M\&Ms) placed at varying distances away, likely requiring fine digit control and reliance on sensory feedback. Second, we wanted to examine reaching for objects with which they had not received any previous experience (e.g., rehabilitative experience). Third, the location of pellets (lateral, rather than in front of the rat) may be sensitive to sensory impairment (i.e., neglect). Two weeks before surgery, animals were trained (two $15 \mathrm{~min}$ trials per day for $12 \mathrm{~d}$ ) to reach for pellets in the staircase test (Montoya et al., 1993). Each staircase consisted of seven steps with three food pellets ( $45 \mathrm{mg}$; Research Diets, New Brunswick, NJ) situated on each step, and the number of pellets eaten per side was used as a measure of forelimb reaching ability. The design of the apparatus prevented any dropped pellets from being retrieved. Because impaired animals may "cheat" by using their tongue to retrieve pellets from the top two steps of the staircase, reaching scores considered only the bottom five steps, from which forelimb use was necessary for successful retrieval. Animals were tested before surgery and then retested for reaching ability at 3 and $4 \mathrm{~d}$ after ischemic injury but before ER treatment. Animals that showed a mild staircase deficit (65-80\% of training performance, which did not meet criteria for ischemic or control groups) were excluded from the experiment. All remaining subjects were retested on days 15-19 and 36-40 after initiation of enriched rehabilitation or social housing (re- 
gardless of start time). Each test period consisted of 10 trials (two trials per day for $5 \mathrm{~d}$ ), and the best three trials were averaged to obtain a maximum level of reaching performance. Three animals did not meet training criterion (minimum of 12 pellets retrieved) and so were excluded from analyses.

Cylinder test of asymmetrical forelimb use. To examine the effect of ischemia and treatment on spontaneous forelimb use during upright postural support movements (Schallert et al., 1997), animals were placed into a clear Plexiglas cylinder $(20 \mathrm{~cm}$ in diameter) situated on a glass tabletop and videotaped from below via an angled mirror. A single cylinder test session was implemented 1 week before ischemia, at $4 \mathrm{~d}$ after ischemia, and again 15 and $36 \mathrm{~d}$ after commencement of treatment. Each session consisted of $5 \mathrm{~min}$ in the cylinder or a minimum of 30 upright wall contacts. The number of forelimb wall contacts (single-limb contacts and bilateral contacts) used for postural support was counted, and the percentage of asymmetry of single-limb wall contacts [(ipsilateral/ ipsilateral + contralateral $) \times 100$ ] was calculated. In addition, the percentage of bilateral limb contacts [bilateral/(ipsilateral + contralateral + bilateral $) \times 100$ ] at each test period was examined separately.

Beam-traversing task. Before surgery, animals were trained for 2 weeks to cross a wooden beam into a darkened plastic tube fixed at the "finish line." The beam was $2.5 \times 180 \mathrm{~cm}$ and was elevated to a height of $1 \mathrm{~m}$ above the floor. Each test session consisted of four trials (two trials in each direction) in which latency to cross the beam and the number of forelimb and hindlimb foot faults were recorded. A fault was defined as any foot slip off the top surface of the beam or any limb use on the side of the beam. Four trials were averaged to give a mean foot fault score, and testing was done 1 week before ischemia, on days 3 or 4 after ischemia, and again at 15 and $36 \mathrm{~d}$ after commencement of treatment.

Ladder-rung walking. The ladder-rung walking test has been reported to be sensitive in detecting long-term impairment in placement, stepping, and coordinated limb use during locomotion after various types of brain injury (Metz and Whishaw, 2002). After 14 and $35 \mathrm{~d}$ of ER, a subset of animals (ER5, $n=5$; ER30, $n=5$; social housing, $n=5$; controls, $n=$ 4) were examined for foot slip and errors in paw placement while traversing a horizontal ladder with variably spaced rungs (Metz and Whishaw, 2002). Unfortunately, this test was not included in our test battery until after the first group of animals had completed treatment (which by chance contained the majority of ER14 animals). Therefore, the ER14 group is not included in the results of this task. This test has been shown recently to be highly sensitive in assessing long-term forelimb and hindlimb sensorimotor function and coordination as well as detecting compensatory movement strategies (Metz and Whishaw, 2002). Because animals had been trained in beam walking before surgery, extensive training was not required to traverse the ladder. Before being tested, animals were given two practice trials on an evenly spaced pattern of rungs and then tested (four trials, twice in each direction) on an irregular pattern of rungs that was varied for each test session. A $60 \mathrm{~cm}$ segment of the ladder was video recorded and used for analysis of foot slips and placement errors. Using a modification of a scoring system that has been described previously (Metz and Whishaw, 2002), errors per step were quantified for each of four trials and averaged for analysis. Forelimb and hindlimb errors resulting in a limb falling through the plane of the bars (misses, deep slips, or slight slips) were counted. Steps precluding and following a stop were not counted. Errors were combined and expressed as the number of errors per step and were averaged over four trials.

In addition, forelimb and hindpaw placement errors that did not result in a slip were also examined. These consisted of replacement, correction, or misplacement of the paw on the rung. The three types of paw placement errors were combined, and placement error per step was averaged over four trials.

\section{Neuroanatomical procedures}

Animals were transcardially perfused with $0.9 \%$ saline, and brains were removed. Whole brains were immersed in a modified Golgi-Cox solution (Glaser and Van der Loos, 1981) for a period of $14 \mathrm{~d}$ before being placed in a $30 \%$ sucrose solution for $2 \mathrm{~d}$. Brains were sectioned at $250 \mu \mathrm{m}$ with a vibratome, slide mounted on $2 \%$ gelatin-coated slides, and stained according to methods described previously (Gibb and Kolb, 1998). Five animals were excluded from anatomical analyses because of poor staining quality (ER5, $n=5$; ER14, $n=7$; ER30, $n=7$; social housing, $n=8$; controls, $n=7)$. Before examination, slides were coded to conceal experimental condition.

Dendritic analyses. Neuronal reconstructions were done as described previously (Biernaskie and Corbett, 2001). Briefly, apical and basilar dendrites of layer $\mathrm{V}$ pyramidal cells located within the forelimb motor cortex (Neafsey et al., 1986) of the undamaged hemisphere were located and drawn at $250 \times$ magnification using the Neurolucida neuron tracing system (Microbrightfield, Colchester, VT). Five cells were drawn for each animal, and the apical and basilar dendritic trees were examined for: (1) total dendritic length, (2) total number of branch segments, (3) centrifugal branch ordering (Coleman and Riesen, 1968), and (4) spine density. To be included in the study, pyramidal cells had to be completely impregnated, visible in the plane of section, and unobstructed by other dendrites, glial cells, or blood vessels.

Spine densities. Dendritic spine densities were examined within the forelimb motor cortex (layer $\mathrm{V}$ pyramidal cells) and dorsolateral striatum from the intact hemisphere. From each reconstructed layer $\mathrm{V}$ pyramidal cell, one terminal basilar (third order or greater) and one terminal apical branch segment from the lower half of the apical tree were drawn. From the dorsolateral striatum, seven terminal branches (each from different medium spiny neurons) were examined. To maintain consistency, each dendritic branch was reconstructed in its entirety (between 50 and $100 \mu \mathrm{m}$ in length) except for approximately the first $10 \mu \mathrm{m}$ (from the last bifurcation) and the last $10 \mu \mathrm{m}$ from the terminal tip. Pyramidal cell reconstructions and dendritic segments were then analyzed using NeuroExplorer software (Microbrightfield). For striatal spine densities, seven animals were excluded from anatomical analyses because of poor staining quality (ER5, $n=5$; ER14, $n=5$; ER30, $n=7$; social housing, $n=8$; controls, $n=7)$.

Infarct measurement. Every third Golgi-stained section through the extent of the brain was examined microscopically to determine regions of injury. Tissue was considered damaged when there was an absence of neuronal cell bodies or dendritic processes. Sections were digitized, and the area of (intact) remaining cortical and striatal tissue (caudate-putamen) from each hemisphere was traced using NIH Image software. The percentage of remaining cortical and striatal tissue within the ischemic hemisphere (relative to the nonischemic hemisphere) was calculated for each brain.

\section{Statistics}

All behavioral data were analyzed using repeated-measures ANOVA or two-factor ANOVA where appropriate. Dendritic arbors and spine densities were analyzed by averaging all cells per animal (or branches for spine densities). ANOVA analyses were used to ascertain effects of ischemic injury or ischemic injury combined with ER (regardless of start time) on dendritic morphology relative to controls. Group differences were ascertained using Fisher's PLSD post hoc comparisons where appropriate. Where indicated, specific planned comparisons were made using unpaired Student's $t$ tests to determine an effect of ER versus social housing. A $p$ value of $<0.05$ was considered significant.

\section{Results}

\section{Infarct measurement}

Figure 1 depicts the areas typically damaged after ET-1-induced MCA occlusion. ANOVA did not show any effect of treatment on the amount of remaining striatal $\left(F_{(3,26)}=0.332 ; p=0.802\right)$, $\operatorname{cortical}\left(F_{(3,26)}=0.402 ; p=0.753\right)$, or total (cortex and striatum) remaining $\left(F_{(3,26)}=0.157 ; p=0.924\right)$ tissue. Mean values of the percentage of remaining cortical or striatal tissue for each group are shown in Table 1. Examination of actual infarct sizes (as opposed to percentages of intact hemisphere) also showed no group differences in size of injury (data not shown).

\section{Single-pellet reaching}

Figure $2 \mathrm{~A}$ depicts a rat in the single-pellet reaching task. Animals were presented with 25 food pellets, and the number of successful 


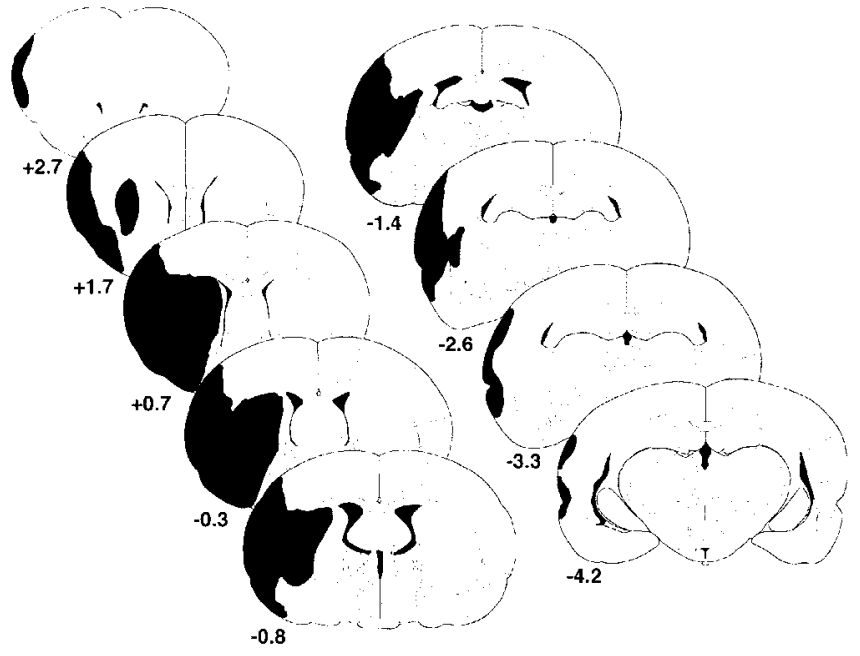

Figure 1. Typical areas of infarction resulting from endothelin-1-induced middle cerebral artery occlusion. Coordinates are relative to bregma (in millimeters). Adapted from Paxinos and Watson (1997).

Table 1. Percentage volume of remaining tissue compared with the undamaged hemisphere

\begin{tabular}{lllll}
\hline & ER5 & ER14 & ER30 & Social housing \\
\hline Cortex & $61.8 \pm 6.6$ & $68.3 \pm 7.7$ & $56.1 \pm 9.78$ & $63.1 \pm 6.4$ \\
Striatum & $42.3 \pm 14.9$ & $30.6 \pm 6.3$ & $33.6 \pm 10.5$ & $29.7 \pm 5.7$
\end{tabular}

Percentage volume of remaining tissue did not statistically differ between various treatment groups. Data are means \pm SE.

reaches and the percentage of accuracy were determined. Figure $2 B$ shows the results of successful reaches over the course of the experiment. Repeated-measures ANOVA confirmed a significant effect of both treatment group $\left(F_{(4,34)}=12.01 ; p<0.0001\right)$ and day $\left(F_{(3,102)}=77.98 ; p<0.0001\right)$ as well as a group by day interaction $\left(F_{(12,102)}=4.55 ; p<0.0001\right)$. All animals were comparable in their ability to retrieve pellets before ischemia, but MCA occlusion resulted in a marked impairment in both number of successful retrievals $(p<0.001)$ and accuracy $(p<0.001)$. Control animals remained consistent throughout the duration of the experiment and performed significantly better than ischemic animals (regardless of treatment) at all time points $(p<0.05)$. No differences were observed between treatment groups at $4 \mathrm{~d}$ after ischemia (i.e., before treatment; $p=0.46$ ). MCA occlusion produced various impairments in reaching. Although qualitative assessment was not done, ischemic injury typically resulted in a postural shift and impairments in limb extension (resulting in many shortened reaches), aim, and supination-pronation of the paw during grasping and release of the pellet into the mouth. In addition, animals frequently exhibited sensory impairments, whereby the animal chased the pellet with its snout after successful retrieval. Animals also successfully grasped a pellet and retracted the paw, but rather than transporting the pellet to the mouth, subsequently placed the paw back on the floor of the cage for support while still clutching the pellet (seemingly unaware of the pellet within the paw). Several animals from each ischemic group exhibited such sensory abnormalities that persisted for the duration of the experiment regardless of treatment.

Skilled reaching ability was assessed again after 14 and $35 \mathrm{~d}$ of social housing or after ER initiated at 5, 14, or $30 \mathrm{~d}$ after stroke. After $14 \mathrm{~d}$, all groups showed slight but nonsignificant improvements in the number of successful reaches. However, by $35 \mathrm{~d}$ after treatment, both ER5 and ER14 animals improved further and retrieved significantly more pellets than ischemic animals exposed to social housing only $(p<0.01)$. In contrast, animals beginning therapy at 1 month (ER30) did not improve to the same extent and were similar to socially housed animals. In fact, ER30 animals retrieved significantly fewer pellets than ER5 animals $(p<0.05)$ when tested after 5 weeks of therapy.

Treatment efficacy was further discriminated by examining reaching accuracy, which accounts for the number of reaching attempts an animal makes to retrieve each pellet (Fig. 2C). Repeated-measures ANOVA revealed an effect of treatment group $\left(F_{(4,34)}=9.789 ; p<0.0001\right)$ and day $\left(F_{(3,102)}=11.114\right.$; $p<0.0001)$, but group by day interaction was not significant $\left(F_{(12,102)}=2.955 ; p=0.0665\right)$. As expected, ischemic injury severely reduced accuracy in skilled reaching compared with control animals before treatment $(p<0.0001)$. Most ischemic animals could not extend the limb far enough to make contact with the pellet, resulting in a series of shortened reaches or additional attempts as a result of aiming errors. Exposure to enriched rehabilitation for $14 \mathrm{~d}$, regardless of start time, did not improve reaching accuracy. However, examination after $35 \mathrm{~d}$ revealed that ER5 animals showed marked functional gains compared with both ER30 and social-housing animals $(p<0.05)$. Thus, ER5 animals were able to obtain more pellets and do so in a more efficient manner (i.e., with fewer attempts). ER14 animals also showed a strong trend for improved accuracy relative to social-housing $(p=0.09)$ animals. By delaying the start of rehabilitation, the effectiveness of rehabilitation was diminished such that ER30 animals were only slightly more accurate than socially housed animals.

\section{Staircase reaching test}

The staircase test showed a significant effect of treatment group $\left(F_{(4,32)}=16.64 ; p<0.001\right)$, an effect of day $\left(F_{(3,12)}=64.26 ; p<\right.$ $0.001)$, and an interaction of treatment group and test day $\left(F_{(3,96)}=6.53 ; p=0.001\right)$. Ischemic injury resulted in a significant reduction in pellets retrieved compared with controls before treatment $(p<0.0001)$, but there was no difference between ischemic groups $(p>0.05)$. As depicted in Figure 3, post hoc analyses revealed no group differences after $14 \mathrm{~d}$ of ER $(p>$ 0.05). However, after $35 \mathrm{~d}$ of therapy, ER5 animals were able to retrieve a greater numbers of pellets relative to socially housed animals $(p<0.05)$ and also showed a strong trend relative to ER30 animals $(p=0.08)$. ER14 was not statistically different compared with ER30 or social housing only.

\section{Beam-walking test}

Figure 4 illustrates the mean numbers of foot faults (slips and placement errors combined) when traversing an elevated narrow beam. Repeated-measures ANOVA showed a significant effect of condition $\left(F_{(4,36)}=4.628 ; p<0.005\right)$ and of day $\left(F_{(3,108)}=31.40\right.$; $p<0.0001)$ as well as a condition by day interaction $\left(F_{(12,108)}=\right.$ $2.471 ; p<0.007)$. Ischemic injury resulted in a significant impairment in all groups relative to controls $(p<0.05)$. At $14 \mathrm{~d}$, a pronounced improvement was observed in ER5 and ER14 animals $(p<0.05)$, and a trend for improvement was observed in ER30 animals $(p=0.08)$ relative to socially housed animals. This improvement persisted in ER5 and ER14 animals after $35 \mathrm{~d}$ of therapy, whereas ER30 animals declined in performance (different from ER14 animals and controls; $p<0.05$ ). Social housing provided minimal recovery, and these animals remained significantly impaired relative to controls at all poststroke time points $(p<0.05)$. 

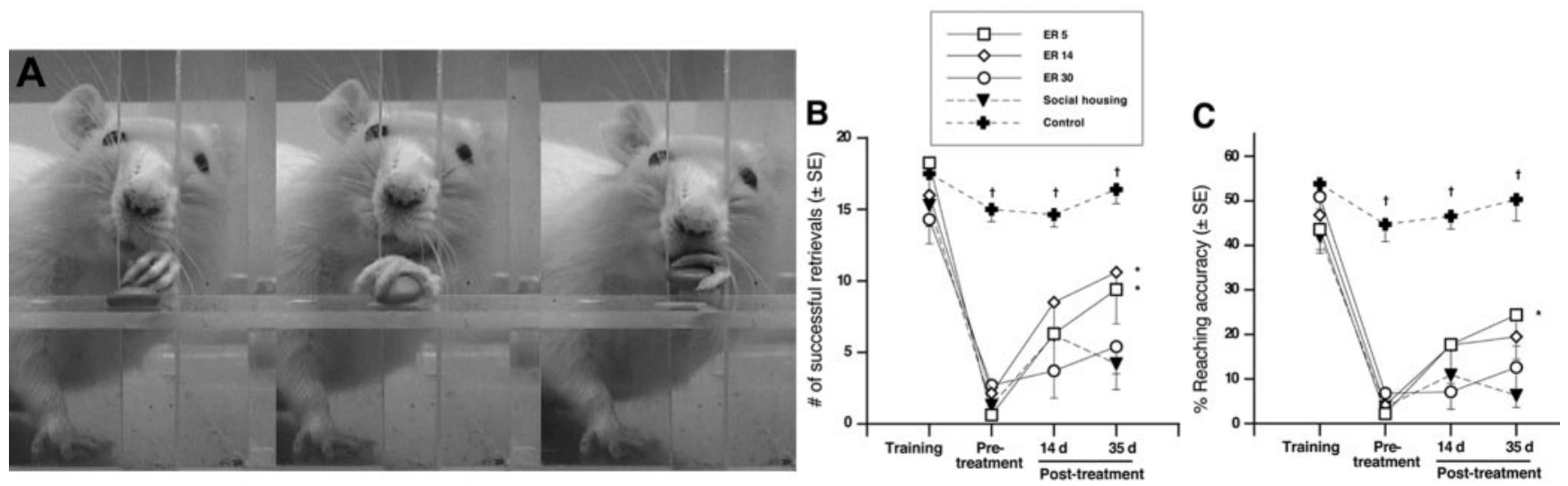

Figure 2. Single-pellet reaching test. $A$, Sequential frames illustrating a rat retrieving a pellet in the pellet reaching test. $B$, Focal ischemia resulted in a severe and persistent impairment in the ability to retrieve pellets. Although all animals showed slight improvement after $14 \mathrm{~d}$ of therapy, by $35 \mathrm{~d}$, ER5 and ER14 animals retrieved significantly more pellets relative to socially housed animals. C, Treatment efficacy was discriminated further by reaching accuracy (which accounts for the number of attempted reaches for each pellet), demonstrating that ER5 animals were improved relative to both ER30 and socially housed animals. ER14 animals showed moderate improvement but did not attain statistical significance $(p=0.09)$. ER5, $n=8 ;$ ER14, $n=7 ;$ ER30, $n=7 ;$ social housing, $n=9$; controls, $n=8 .{ }^{*} p<0.05$ from socially housed animals; ${ }^{\dagger} p<0.001$ from controls.

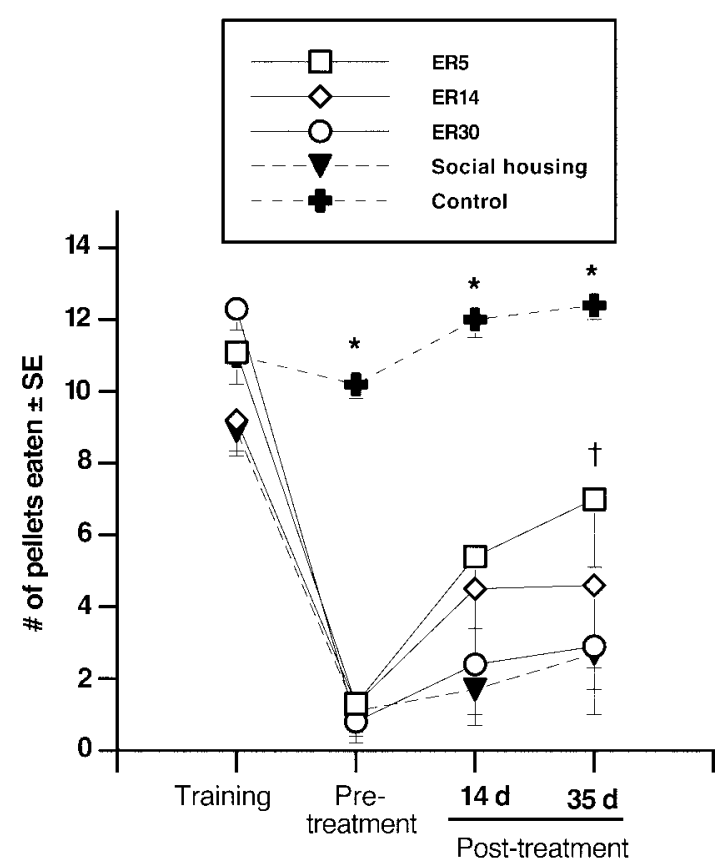

Figure 3. Staircase reaching test. The mean number of pellets eaten from the staircase below the impaired limb is shown. Because rats were able to lick pellets from the top two steps, reaching scores only included the bottom five steps of the staircase. All ischemic groups were severely impaired on days 3 and 4 after ischemia (before treatment; $p<0.001$ ) relative to controls. Although there was no difference between ischemic groups before treatment or after $14 \mathrm{~d}$ of respective treatment ( $p>0.05)$, ER5 animals were able to retrieve more pellets than socially housed animals after $35 \mathrm{~d}$ of treatment $(p<0.05)$. ER5 animals also showed a strong trend for improvement relative to ER30 animals ( $p=0.07)$. ER5, $n=8 ; \mathrm{ER} 14, n=7$; ER30, $n=7$; social housing, $n=6$; controls, $n=9$. ${ }^{*} p<0.05$ different from all other groups; ${ }^{\dagger} p<$ 0.05 different from social housing.

\section{Cylinder test}

Animals did not exhibit asymmetrical use of the forelimbs during postural support before ischemic injury. After surgery, all ischemic animals developed a reliance on the ipsilateral limb for postural support that persisted for the duration of the experiment. For single-limb contacts (Fig. 5A), repeated-measures ANOVA verified a main effect of group $\left(F_{(4,36)}=6.743 ; p<0.005\right)$, day $\left(F_{(3,108)}=30.03 ; p<0.0001\right)$, and group by day interaction

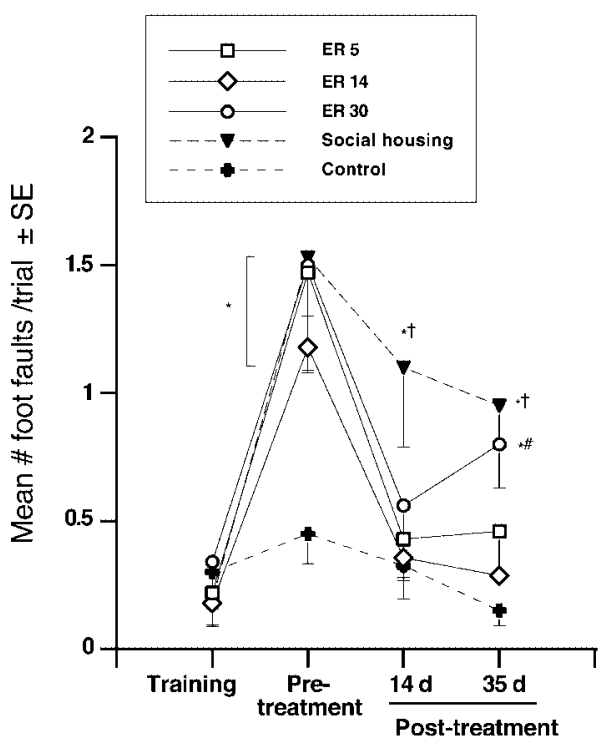

Figure 4. Narrow-beam-walking task. Four days after ischemia (before treatment), all groups made a greater number of foot slips relative to controls $(p<0.01)$. ER provided a marked reduction in the number of foot faults when initiated at either 5 or $14 \mathrm{~d}$ after the stroke. After 14d, ER (regardless of start time) reduced foot slips compared with social housing, and this improvement persisted after $35 \mathrm{~d}$ in ER5 and ER14 animals. ER5, $n=8$; ER14, $n=7 ;$ ER30, $n=$ 7; social housing, $n=9$; controls, $n=9$. ${ }^{*} p<0.01$, different from controls; ${ }^{\dagger} p<0.05$, different from ER5 and ER14; ${ }^{\#} p<0.01$, different from ER14.

$\left(F_{(12,108)}=3.89 ; p<0.0001\right)$. Surprisingly, this reliance declined in ER5 animals, did not differ from controls after $35 \mathrm{~d}$ of treatment $(p>0.05)$, and was significantly reduced relative to ER30 and social-housing animals after $14(p<0.05)$ but not after $35 \mathrm{~d}$ of treatment. ER14 animals also showed less forelimb asymmetry after $14 \mathrm{~d}$ compared with ER30 animals $(p<0.05)$. ER14, ER30, and socially housed animals were significantly more dependent on the ipsilateral limb at all poststroke test times relative to control animals $(p<0.05)$.

Bilateral limb contacts were also markedly reduced after ischemia (Fig. 5B). Before surgery, $\sim 40-50 \%$ of postural support movements were bilateral, and after ischemia, this declined to $\sim 25 \%$. A significant effect of treatment group $\left(F_{(4,36)}=3.10\right.$; $p<$ $0.03)$ and day $\left(F_{(3,108)}=17.66 ; p<0.0001\right)$ as well as a group by 
day interaction $\left(F_{(12,108)}=1.96 ; p<0.04\right)$ was observed for bilateral limb use. Surprisingly, after $14 \mathrm{~d}$ of therapy, ER5 animals did not differ from control animals, although all other groups made fewer bilateral limb contacts relative to control animals (i.e., $42 \%$ of limb contacts; $p<$ $0.05)$. This same trend continued after $35 \mathrm{~d}$, although only socially housed animals remained different from control animals $(p<0.02)$. These data suggest that early rehabilitative intervention may (to some extent) counteract the learned nonuse of the impaired paw after ischemic stroke.

\section{Ladder-rung walking task}

Figure $6 A$ shows the number of errors per step made while walking across an irregular pattern of metal rungs after 14 and $35 \mathrm{~d}$ of treatment. Repeated-measures ANOVA confirmed an effect of treatment condition $\left(F_{(3,15)}=11.63 ; p<0.001\right)$. Surprisingly, ER5 animals performed comparably to control animals at 14 and $35 \mathrm{~d}$ after treatment. However, despite enriched housing, the error rate for ER30 animals was increased compared with ER5 animals $(p<$ 0.05 ) and approached significance compared with control animals $(p=0.09$ ). Interestingly, by $35 \mathrm{~d}$ after treatment, ER30 animals had recovered to ER5 and control levels. Animals exposed to social housing were significantly impaired relative to both ER5 and control animals $(p<0.05)$ after $14 \mathrm{~d}$, and this impairment persisted after $35 \mathrm{~d}$ of treatment $(p<0.004)$.

Incorrect paw placements that did not result in a slip (i.e., replacements, corrections, and partial placements) were also examined and are shown in Figure $6 B$. These three types of errors were combined for each trial, and the number of errors per step was calculated for each trial and averaged. Repeated-measures ANOVA reflected an effect of group $\left(F_{(3,15)}=4.58 ; p<0.02\right)$ but no effect of day $\left(F_{(1,15)}=1.57 ; p=0.23\right)$ or interaction $\left(F_{(3,15)}=\right.$ $0.80 ; p=0.51)$. After $14 \mathrm{~d}$, ER5 animals performed similarly to control animals $(p=0.56)$ and had significantly decreased error rates compared with ER30 and social-housing animals $(p<$ 0.05). After $35 \mathrm{~d}$ of treatment, all ischemic groups (ER5, ER30, and social-housing animals) had improved. Despite this improvement, ischemic groups still committed more errors relative to controls, but this difference was not significant.

\section{Dendritic morphology within the undamaged motor system} Dendritic morphology of forelimb motor cortex

To examine the effects of early or delayed rehabilitative therapy on neural connectivity after stroke, we examined the dendritic morphology of layer $\mathrm{V}$ pyramidal cells within the forelimb motor cortex of the undamaged hemisphere. These neurons include those that project to the forelimb region of the rat spinal cord (Coleman and Riesen, 1968), striatum (Gerfen, 1989), and red nucleus (Giuffrida et al., 1991), collectively contributing to forelimb movement. Also, they are sensitive to experience in the normal brain (Greenough et al., 1985) and to rehabilitative experience after injury (Jones et al., 1999; Biernaskie and Corbett, 2001).



Figure 6. Ladder-rung walking test. $A$, ER5 animals showed a significant reduction in errors per step while traversing an irregular pattern of rungs compared with both ER30 and socialhousing animals. ER30 resulted in a delayed improvement that reached control levels by $35 \mathrm{~d}$ after treatment. A persistent impairment was observed in socially housed animals. $B$, Foot placement errors were significantly elevated at $14 \mathrm{~d}$ after treatment. After $35 \mathrm{~d}$ of therapy, all ischemic groups made a greater number of placement errors compared with controls, although this did not reach significance. ER5, $n=5$; ER30, $n=5$; social housing, $n=5$; controls, $n=4$. ${ }^{*} p<0.05$ relative to controls; ${ }^{\dagger} p<0.05$ different from ER5; ${ }^{\S} p<0.05$ relative to ER30.

\section{Dendritic length}

Reconstructed cells were measured for total dendritic length, total number of dendritic branches, and complexity of dendritic arbors (branch order). Ischemic injury alone did not significantly 


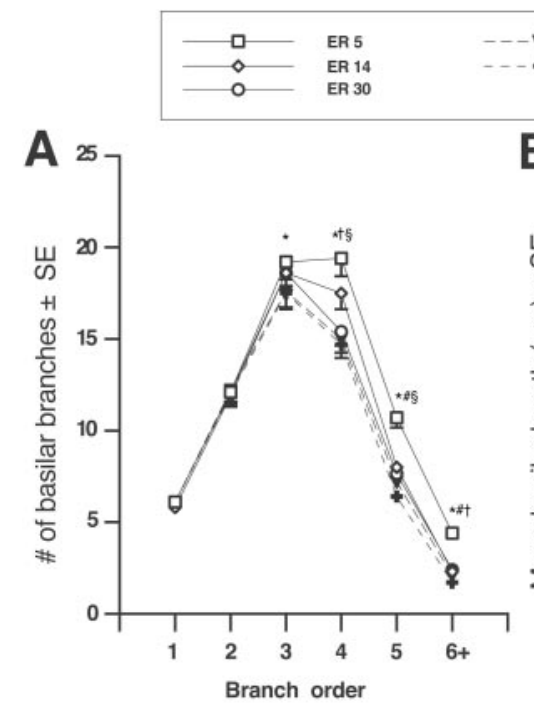

Figure 7. Dendritic branching in the undamaged motor cortex. A, Complexity of the basilar dendritic arbor for layer $V$ pyramidal cells was analyzed by branch order, an indicator of increasing numbers of branch bifurcations from the cell body. ER5 elevated the number of higher-order branches relative to all other groups. ER5 resulted in significant elevations at fourth-order, fifth-order, and sixth-order branches compared with controls. Also, early rehabilitation enhanced the dendritic branch number relative to delayed ER; ER5 was different from ER14 (at fifth- and sixth-order branches) and ER30 (at fourth-, fifth-, and sixth-order branches). B, Total dendritic length per cell in ER5, ER14, and ER30 animals was increased relative to controls; however, there was no change in length resulting from the ischemic insult. ER5, $n=5$; ER14, $n=7$; ER30, $n=7$; social housing, $n=8$; controls, $n=7 .{ }^{*} p<0.05$ from controls; ${ }^{*} p<0.05$ from ER14; ${ }^{\S} p<0.05$ from ER30; ${ }^{\dagger} p<0.05$ from social housing.

alter dendritic length compared with control levels $\left(F_{(1,33)}=\right.$ $2.011 ; p=0.165)$; however, ischemia combined with ER resulted in a significant elevation in dendritic length $\left(F_{(3,22)}=3.85 ; p<\right.$ 0.03 ) compared with controls (Fig. 7B). Post hoc group comparisons confirmed that ER5 $(p<0.005)$ and ER14 $(p<0.02)$ significantly increased basilar length relative to controls. This was not observed after ER30 $(p=0.113)$ or in ischemic animals exposed to social housing ( $p=0.81$ ). Early initiation of ER (ER5) did not increase dendritic length compared with ER14 ( $p=0.50)$ or $\operatorname{ER} 30(p=0.11)$.

\section{Number of dendritic branches}

Ischemic injury alone did not affect the total number of basilar dendritic branches $\left(F_{(1,33)}=2.23 ; p=0.14\right)$, but ER treatment after ischemia did increase branches $\left(F_{(3,22)}=4.224 ; p<0.02\right)$ compared with controls. However, only ER5 produced an increase in the total number of branches relative to controls $(p<$ 0.02). Similarly, ER5 animals showed elevated branches compared with ER30 $(p<0.05)$ and social-housing $\left(t_{(12)}=2.13 ; p<\right.$ $0.05)$ animals. No differences were observed between ER14 and ER30 animals $(p=0.63)$, and delayed ER animals (ER14 or ER30) did not increase the number of dendritic branches compared with social-housing animals $(p>0.69)$.

\section{Dendritic complexity}

Complexity of the dendritic arbor was analyzed using branchorder analysis and is shown in Figure 7A. Ischemic injury did not affect the number of branches at any order $(p>0.20)$ compared with controls; however, ANOVA revealed that ischemia followed by ER resulted in a significant elevation in branches at fourthorder $\left(F_{(3,22)}=3.24 ; p<0.05\right)$, fifth-order $\left(F_{(3,22)}=4.58 ; p<\right.$ $0.02)$, and sixth-order $\left(F_{(3,22)}=2.95 ; p<0.05\right)$ branches. Post hoc
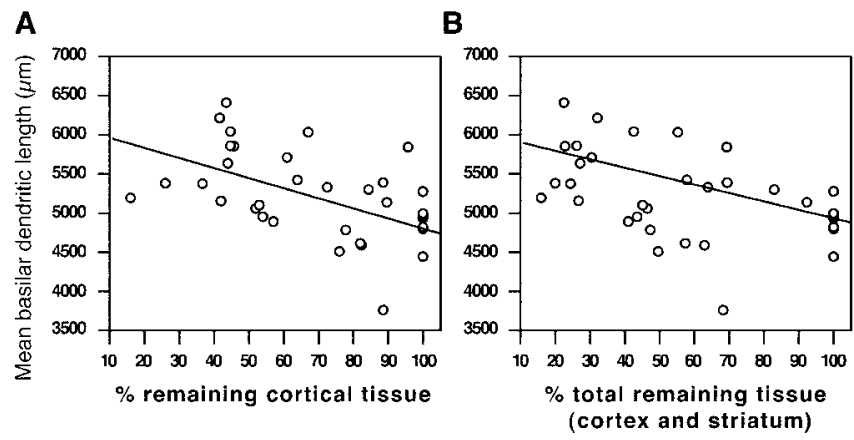

Figure 8. Size of infarction correlates with dendritic length in the undamaged motor cortex. Decreasing amounts of remaining cortical $(A)$ or total $(B)$ tissue (cortical and striatal) after ischemic injury resulted in greater dendritic length of layer $V$ pyramidal cells in the undamaged motor cortex. Cortical tissue, $R^{2}=0.209, p=0.0192$; total remaining tissue, $R^{2}=0.212, p=$ 0.0054 .

group comparisons revealed that ER5 animals had increased fourth-order $(p<0.01)$, fifth-order $(p<0.003)$, and sixthorder $(p<0.01)$ branches compared with control animals. The effect of ER on dendritic growth seemed to be timing dependent, because ER14 and ER30 animals failed to produce a significant change in branch numbers at any order relative to the control group $(p>0.05)$. Indeed, the earliest exposure to ER (ER5) increased fifth-order $(p<0.04)$ and sixth-order $(p<0.03)$ branches relative to ER14. Moreover, a comparison of ER5 with ER30 animals demonstrated a significant increase at fourth-order $(p<0.02)$, fifth-order $(p<0.02)$, and sixth-order $(p<0.05)$ branches. Finally, we also compared the ER5 animals with socially housed animals and found that ER5 was increased at fourth-order $\left(t_{(12)}=2.2 ; p<0.05\right)$ and sixth-order $\left(t_{(12)}=2.6\right.$; $p<0.03$ ) branches. Ischemic animals exposed to social housing did not differ from ER14 or ER30 animals at any branch order.

To summarize, ER after ischemic injury (but not social housing) resulted in enhanced dendritic growth in the undamaged hemisphere. However, ER5 significantly increased dendritic length and branch number relative to ER14 and even further compared with ER30, indicating that the intact motor cortex is more sensitive to rehabilitative experience initiated earlier rather than later after an ischemic insult.

Dendritic growth was also examined in relation to infarct size. Regression analysis revealed a relationship between infarct size and the amount of total dendritic length of layer $\mathrm{V}$ pyramidal cells (cortical tissue, $R^{2}=0.209, p=0.0192$; total remaining tissue, $\left.R^{2}=0.212, p=0.0054\right)$. That is, increasing infarct size resulted in increased dendritic length within the undamaged motor cortex (Fig. 8). This indicates that not only does rehabilitative experience influence neuromorphological remodeling but so does the size or spatial distribution of the ischemic injury itself.

\section{Spine densities}

Layer V pyramidal cells

ANOVA showed no effect of ischemic injury on apical $\left(F_{(4,30)}=\right.$ $0.454 ; p=0.77)$ or basilar $\left(F_{(4,30)}=0.40 ; p=0.81\right)$ spine densities of layer $\mathrm{V}$ pyramidal cells within the intact motor cortex. Similarly, application of ER at any time after ischemic injury failed to produce a significant change in either apical or basilar spine densities $(p>0.05)$. It is worth noting that there appeared to be an abundance of multiheaded spines as well as long thin spines lacking a defined spine head, perhaps indicating morphological spine changes rather than an increase in the number of spines. Nevertheless, the finding that ER5 (and to a lesser extent 
ER14) enhanced dendritic growth of layer V pyramidal cells without a decrease in spine density suggests that new spines are added to these dendritic extensions, ultimately increasing synaptic connectivity within the motor cortex.

\section{Dorsolateral striatum}

Spine densities were also assessed from terminal branches of medium spiny neurons within the dorsolateral striatum of the intact hemisphere. ANOVA confirmed no effect of ischemic injury $\left(F_{(1,30)}=1.31 ; p=0.261\right)$ or ischemia followed by $\operatorname{ER~}\left(F_{(3,22)}=\right.$ $0.777 ; p=0.52)$ on striatal spine densities.

\section{Discussion}

The goal of the present study was to determine whether there exists a critical period after stroke during which the brain is most responsive to rehabilitative therapy. Our results confirm our hypothesis that initiating rehabilitation earlier (e.g., $5 \mathrm{~d}$ ) rather than later (e.g., $30 \mathrm{~d}$ ) provides significantly greater functional compensation-recovery and enhanced structural plasticity within the undamaged motor cortex.

\section{Earlier initiation enhances efficacy of rehabilitation}

Our findings are supported by previous work (Barbay et al., 2001) demonstrating a time-dependent, rehabilitation-induced map reorganization after ischemic injury in primates. Earlier rehabilitation resulted in greater preservation of forelimb cortical maps compared with similar treatment initiated later. This is consistent with the idea that the remaining cortical tissues (ipsilateral and contralateral to the infarct) are most responsive to rehabilitative training early after a stroke, and that this effect declines with time. Our results demonstrate the functional consequence of this sensitivity. ER5 animals were significantly improved in skilled forelimb reaching and in tests of coordinated forelimb use during locomotor activity (narrow-beam and ladder-rung walking) relative to animals that received the same therapy beginning 1 month later. ER5 animals also showed qualitative gains such that reaching accuracy and affected-limb use during postural support were both improved. In contrast, ER30 animals did not differ from social-housing animals on any task, with the exception of ladder-rung walking, although this improvement was delayed relative to ER5. It is also noteworthy that shorter delays to therapy (e.g., $14 \mathrm{~d}$ ) provided moderate functional gains in reaching ability and beam walking compared with social housing.

\section{Early rehabilitation (ER5) does not exacerbate infarct size or behavioral impairment}

Several previous studies noted an exacerbation of injury and worsened overall outcome after excessive use of the impaired limbs (i.e., by casting or enrichment) beginning immediately after the injury (Kozlowski et al., 1996; Risedal et al., 1999). Other studies report that despite exacerbation of ischemic injury, immediate exposure to environmental enrichment improves functional outcome (Risedal et al., 1999; Farrell et al., 2001), perhaps as a result of removing functionally abnormal neurons. Here we failed to observe any exacerbation of injury after ER5, and recovery was maximized when compared with ER14 and ER30. During the first week after injury, tissue surrounding the infarct becomes hyperexcitable (Neumann Haefelin and Witte, 2000). Excessive sensorimotor activation too early after the insult may exacerbate injury through a use-dependent, NMDA-mediated process (Humm et al., 1999). The virulence of this process may dissipate over days, explaining why rehabilitative experience (i.e., acrobatic or reach training) beginning 3-5 d after insult does not worsen injury size or behavioral outcome (Nudo et al., 1996; Jones et al., 1999).

\section{Dendritic morphology within the undamaged hemisphere}

ER initiated early after ischemic stroke resulted in a marked increase in dendritic growth and complexity that corresponded to improved function of the affected forelimb and hindlimb not observed in ER30 and socially housed animals. The amount of dendritic growth in the undamaged motor cortex was associated with both lesion size and improved functional outcome. Neuronal reorganization within this region likely reflects unmasking and reorganization of ipsilateral corticospinal projections (Lee and van Donkelaar, 1995) or axonal sprouting and formation of novel subcortical projections (Kawamata et al., 1997; Chen et al., 2002; Papadopoulos et al., 2002). Although it is conceivable that recovery could be supported by the minimal ipsilateral (uncrossed) projections from the undamaged motor cortex to cervical spinal cord, it is more likely that the corticofugal sprouting from the undamaged motor cortex to the red nucleus (on the affected side) is contributing to improved function (Chen et al., 2002; Papadopoulos et al., 2002). Indeed, Emerick et al. (2003) showed that after cortical injury combined with IN-1 (an antibody against Nogo-A) infusion, microstimulation of the undamaged motor cortex evoked a greater number of movements of the ipsilateral (affected) forepaw. Furthermore, functional imaging studies in human stroke subjects report altered patterns of activation in both the damaged and undamaged motor cortices during movement of the affected limb (Cramer et al., 1997; Cuadrado et al., 1999; Kopp et al., 1999; Feydy et al., 2002; Johansen-Berg et al., 2002). Moreover, in recovering stroke patients, Johansen-Berg et al. (2002) showed that disruption of ipsilateral motor activation using transcranial motor stimulation reduced reaction times in a finger movement task, providing direct evidence for an expanding functional role of the undamaged motor system after severe ischemic infarction.

An important finding was that greater tissue infarction produced more dendritic growth in the undamaged motor cortex, indicating that the pattern of neuronal reorganization after ischemia seems to be dependent on the size and distribution of the injury. This is consistent with a recent longitudinal clinical study reporting that if the primary motor cortex (M1) was intact, recruitment of the undamaged hemisphere was transient, whereas if M1 was damaged, compensatory recruitment of the undamaged hemisphere persisted (e.g., sensorimotor cortex, premotor area) (Feydy et al., 2002). In some cases, infarct size may also be reflected by functional impairment. It has been suggested that the incidence of bilateral motor activation is dependent on initial impairment (Johansen-Berg et al., 2002; for review, see Calautti and Baron, 2003). Small cortical lesions may induce remodeling in adjacent regions, which possess similar projection patterns and thus more efficiently compensate for impaired function. In contrast, large infarcts affecting multiple structures may involve more widespread reorganization in adjacent and remote regions (i.e., the undamaged hemisphere), recruiting less efficient projections and ultimately limiting functional recovery. Interestingly, most human imaging studies demonstrate that in patients with the greatest functional recovery, movement-induced activation patterns have returned to the affected hemisphere (contralateral to the impaired limb) (Calautti and Baron, 2003). However, with increasing infarct size, reinstatement of tissues surrounding the infarct may not be possible, thus resulting in persistent activation of the undamaged hemisphere. The ER-induced dendritic growth observed within the undamaged motor system of ER5 
animals may reflect an enhanced plasticity, supporting persistent recruitment, or alternatively may contribute to a transient but necessary process, leading to the reorganization and subsequent reinstatement of viable tissues within the affected hemisphere (Calautti and Baron, 2003).

Despite the moderate improvement provided by ER14, only dendritic length (not complexity) was significantly increased relative to control animals, as it had been in our previous study (Biernaskie and Corbett, 2001). Any delay (relative to ER5) may dampen the efficacy of rehabilitation, thereby requiring longer duration or more intensive therapy. Our original study applied 9 weeks of ER, as opposed to 5 weeks here, which may account for the lessened functional gains and dendritic plasticity observed in the latter. Furthermore, both control and no treatment groups differed in the two studies. In our previous study, both ischemic animals receiving no treatment and control animals were singly housed, whereas social housing was used here. Social housing has been shown to produce changes in brain morphology (Connor et al., 1982) and improve recovery (Risedal et al., 2002) relative to isolated animals. It is possible that the social-housing conditions implemented here may have enhanced dendritic complexity, thereby reducing the anatomical differences between ER14 and untreated ischemic animals observed in our previous study (Biernaskie and Corbett, 2001).

Increased spine densities have been associated with greater synaptic connectivity and enhanced functional recovery (Kolb et al., 1997). Despite the behavioral improvement and dendritic growth observed in ER5 animals, there was no change in layer $\mathrm{V}$ spine densities. Similarly, Johansson and Belichenko (2002) reported that environmental enrichment elevated layer $\mathrm{V}$ spines in normal animals but not when enrichment was applied after MCA occlusion. Interestingly, enrichment after ischemic injury did elevate spine densities in layers II and III (Belichenko et al., 2001). Together, our data suggest that early ER after ischemic injury enhances dendritic growth of layer V neurons; because the additional dendritic material maintains a similar spine density, the net effect would be an increase in the number of spines per cell and ultimately greater synaptic connectivity. The observed changes also support the idea of laminar-specific morphological plasticity after either injury or behavioral experience (Adkins et al., 2002). Unfortunately, in this study, examination of individual spine morphologies was not possible. Finally, although spine densities remained constant, it is also possible that the distribution of transient versus stable spines (Trachtenberg et al., 2002) was altered by ischemic injury and ER and should be addressed in future studies.

\section{Potential mechanisms shaping the critical period}

Transient expression of various neurotrophic factors may determine the boundaries of this critical period. In nonischemic regions, bFGF (Speliotes et al., 1996) and NGF mRNA are transiently altered after focal ischemia and can be influenced further by behavioral experience (Humm et al., 1997; Dahlqvist et al., 1999). For example, restricting use of the impaired forelimb after cortical injury inhibited astrocytic expression of bFGF in surrounding tissues (Humm et al., 1997), suggesting that activation of the compromised motor system is critical for expression of growth-promoting molecules.

The critical period may also be defined by the early hyperexcitability that occurs in tissues ipsilateral and contralateral to the ischemic infarct, which subsequently dissipates over several weeks (Buchkremer-Ratzmann et al., 1996; Neumann Haefelin and Witte, 2000). Increased excitability coincides with the period during which commencement of ER produces greatest recovery. Pharmacological prevention of hyperexcitability during the first 3 weeks after brain injury (but not later) delays recovery (Schallert et al., 1986), suggesting that this excitable state may support processes of recovery.

In summary, our results suggest that the poststroke brain is in a state of heightened sensitivity to behavioral experience reminiscent of the critical periods during development of the visual system (Hubel et al., 1977). Initiating rehabilitative therapy early after the stroke provided significant functional gains and enhanced structural plasticity relative to the same treatment delayed by 1 month. This suggests that delaying commencement of rehabilitation may reduce the efficacy of treatment, ultimately limiting functional recovery in stroke patients.

\section{References}

Adkins DL, Bury SD, Jones TA (2002) Laminar-dependent dendritic spine alterations in the motor cortex of adult rats following callosal transection and forced forelimb use. Neurobiol Learn Mem 78:35-52.

Barbay S, Plautz EP, Friel KM, Frost SB, Stowe AM, Dancause N, Wang H, Nudo RJ (2001) Delayed rehabilitative training following a small ischemic infarct in nonhuman primate primary motor cortex (M1). Soc Neurosci Abstr 27:931.4.

Belichenko PV, Mattsson B, Johansson BB (2001) Neuronal and fibre organization in neocortical grafts placed in post-ischaemic adult rat brain: a three-dimensional confocal microscopy study. J Comp Pathol 124:142-148.

Biernaskie J, Corbett D (2001) Enriched rehabilitative training promotes improved forelimb motor function and enhanced dendritic growth following focal ischemic injury. J Neurosci 21:5272-5280.

Biernaskie J, Corbett D, Peeling J, Wells J, Lei H (2001) A serial MR study of cerebral blood flow changes and lesion development following endothelin-1-induced ischemia in rats. Magn Reson Med 46:827-830.

Buchkremer-Ratzmann I, August M, Hagemann G, Witte OW (1996) Electrophysiological transcortical diaschisis after cortical photothrombosis in rat brain. Stroke 27:1105-1111.

Calautti C, Baron J-C (2003) Functional neuroimaging studies of motor recovery after stroke in adults: a review. Stroke 34:1553-1566.

Chen P, Goldberg DE, Kolb B, Lanser M, Benowitz LI (2002) Inosine induces axonal rewiring and improves behavioral outcome after stroke. Proc Natl Acad Sci USA 99:9031-9036.

Coleman PD, Riesen AH (1968) Environmental effects on cortical dendritic fields. I. Rearing in the dark. J Anat 102:363-374.

Connor JR, Beban SE, Melone JH, Yuen A, Diamond MC (1982) A quantitative Golgi study in the occipital cortex of the pyramidal dendritic topology of old adult rats from social or isolated environments. Brain Res 251:39-44.

Cramer SC, Nelles G, Benson RR, Kaplan JD, Parker RA, Kwong KK, Kennedy DN, Finklestein SP, Rosen BR (1997) A functional MRI study of subjects recovered from hemiparetic stroke. Stroke 28:2518-2527.

Cuadrado ML, Egido JA, Gonzalez-Gutierrez JL, Varela-De-Seijas E (1999) Bihemispheric contribution to motor recovery after stroke: a longitudinal study with transcranial Doppler ultrasonography. Cerebrovasc Dis 9:337-344.

Dahlqvist P, Zhao L, Johansson IM, Mattsson B, Johansson BB (1999) Environmental enrichment alters nerve growth factor-induced gene $\mathrm{A}$ and glucocorticoid receptor messenger RNA expression after middle cerebral artery occlusion in rats. Neuroscience 93:527-535.

Emerick AJ, Neafsey EJ, Schwab ME, Kartje GL (2003) Functional reorganization of the motor cortex in adult rats after cortical lesion and treatment with monoclonal antibody IN-1. J Neurosci 23:4826-4830.

Farrell R, Evans S, Corbett D (2001) Environmental enrichment enhances recovery of function but exacerbates ischemic cell death. Neuroscience 107:585-592.

Feydy A, Carlier R, Roby-Brami A, Bussel B, Cazalis F, Pierot L, Burnod Y, Maier MA (2002) Longitudinal study of motor recovery after stroke: recruitment and focusing of brain activation. Stroke 33:1610-1617.

Gerfen CR (1989) The neostriatal mosaic: striatal patch-matrix organization is related to cortical lamination. Science 246:385-388. 
Gibb R, Kolb B (1998) A method for vibratome sectioning of Golgi-Cox stained whole rat brain. J Neurosci Methods 79:1-4.

Giuffrida R, Aicardi G, Rapisarda C (1991) Projections from the cerebral cortex to the red nucleus in the guinea pig: a retrograde tracing study. Eur J Neurosci 3:866-875.

Glaser EM, Van der Loos H (1981) Analysis of thick brain sections by obverse-reverse computer microscopy: application of a new, high clarity Golgi-Nissl stain. J Neurosci Methods 4:117-125.

Greenough WT, Larson JR, Withers GS (1985) Effects of unilateral and bilateral training in a reaching task on dendritic branching of neurons in the rat motor-sensory forelimb cortex. Behav Neural Biol 44:301-314.

Hubel DH, Wiesel TN, Levay S (1977) Plasticity of ocular dominance columns in monkey striate cortex. Philos Trans R Soc Lond B Biol Sci 278:377-409.

Humm JL, James DC, Gibb R, Kolb B, Schallert T (1997) Forced disuse of the impaired forelimb by restraint after sensorimotor cortical injury inhibits expression of endogenous bFGF in astrocytes and adversely affects behavioral function. Soc Neurosci Abstr 23:95.95.

Humm JL, Kozlowski DA, Bland ST, James DC, Schallert T (1999) Usedependent exaggeration of brain injury: is glutamate involved? Exp Neurol 157:349-358

Johansen-Berg H, Rushworth MFS, Bogdanovic MD, Kischka U, Wimalaratna S, Matthews PM (2002) The role of the ipsilateral premotor cortex in hand movement after stroke. Proc Natl Acad Sci USA 99:14518-14523.

Johansson BB, Belichenko PV (2002) Neuronal plasticity and dendritic spines: effect of environmental enrichment on intact and postischemic rat brain. J Cereb Blood Flow Metab 22:89-96.

Jones TA, Chu CJ, Grande LA, Gregory AD (1999) Motor skills training enhances lesion-induced structural plasticity in the motor cortex of adult rats. J Neurosci 19:10153-10163.

Kawamata T, Alexis NE, Dietrich WD, Finklestein SP (1996) Intracisternal basic fibroblast growth factor enhances behavioral recovery following focal cerebral infarction in the rat. J Cereb Blood Flow Metab 16:542-547.

Kawamata T, Dietrich WD, Schallert T, Gotts JE, Cocke RR, Benowitz LI, Finklestein SP (1997) Intracisternal basic fibroblast growth factor enhances functional recovery and up-regulates the expression of a molecular marker of neuronal sprouting following focal cerebral infarction. Proc Natl Acad Sci USA 94:8179-8184.

Kolb B, Stewart J, Sutherland RJ (1997) Recovery of function is associated with increased spine density in cortical pyramidal cells after frontal lesions and/or noradrenaline depletion in neonatal rats. Behav Brain Res 89:61-70.

Kopp B, Kunkel A, Muhlnickel W, Villringer K, Taub E, Flor H (1999) Plasticity in the motor system related to therapy-induced improvement of movement after stroke. NeuroReport 10:807-810.

Kozlowski DA, James DC, Schallert T (1996) Use-dependent exaggeration of neuronal injury after unilateral sensorimotor cortex lesions. J Neurosci 16:4776-4786.

Lee RG, van Donkelaar P (1995) Mechanisms underlying functional recovery following stroke. Can J Neurol Sci 22:257-263.

McNeill TH, Mori N, Cheng HW (1999) Differential regulation of the growth-associated proteins, GAP-43 and SCG-10, in response to unilateral cortical ablation in adult rats. Neuroscience 90:1349-1360.

Metz GA, Whishaw IQ (2002) Cortical and subcortical lesions impair skilled walking in the ladder rung walking test: a new task to evaluate foreand hindlimb stepping, placing, and co-ordination. J Neurosci Methods 115:169-179.

Montoya CP, Campbell-Hope LJ, Pemberton KD, Dunnett SB (1993) The "staircase test": a measure of independent forelimb reaching and grasping abilities in rats. J Neurosci Methods 36:219-228.

Neafsey EJ, Bold EL, Haas G, Hurley-Gius KM, Quirk G, Sievert CF, Terreberry RR (1986) The organization of the rat motor cortex: a microstimulation mapping study. Brain Res 396:77-96.

Neumann Haefelin T, Witte OW (2000) Periinfarct and remote excitability changes after transient middle cerebral artery occlusion. J Cereb Blood Flow Metab 20:45-52.

Nudo RJ, Wise BM, SiFuentes F, Milliken GW (1996) Neural substrates for the effects of rehabilitative training on motor recovery after ischemic infarct. Science 272:1791-1794.

Papadopoulos CM, Tsai SY, Alsbiei T, O’Brien TE, Schwab ME, Kartje GL (2002) Functional recovery and neuroanatomical plasticity following middle cerebral artery occlusion and IN-1 antibody treatment in the adult rat. Ann Neurol 51:433-441.

Paxinos G, Watson C (1997) The rat brain in stereotaxic coordinates. San Diego: Academic.

Peterson GM, Devine JV (1963) Transfer on handedness in the rat resulting from small cortical lesions after limited forced practice. J Comp Physiol Psychol 56:752-756.

Risedal A, Zeng J, Johansson BB (1999) Early training may exacerbate brain damage after focal brain ischemia in the rat. J Cereb Blood Flow Metab 19:997-1003.

Risedal A, Mattsson B, Dahlqvist P, Nordborg C, Olsson T, Johansson BB (2002) Environmental influences on functional outcome after a cortical infarct in the rat. Brain Res Bull 58:315-321.

Schallert T, Hernandez TD, Barth TM (1986) Recovery of function after brain damage: severe and chronic disruption by diazepam. Brain Res 379:104-111.

Schallert T, Kozlowski DA, Humm JL, Cocke RR (1997) Use-dependent structural events in recovery of function. Adv Neurol 73:229-238.

Sharkey J, Ritchie IM, Kelly PA (1993) Perivascular microapplication of endothelin-1: a new model of focal cerebral ischaemia in the rat. J Cereb Blood Flow Metab 13:865-871.

Speliotes EK, Caday CG, Do T, Weise J, Kowall NW, Finklestein SP (1996) Increased expression of basic fibroblast growth factor (bFGF) following focal cerebral infarction in the rat. Brain Res Mol Brain Res 39:31-42.

Stroemer RP, Kent TA, Hulsebosch CE (1995) Neocortical neural sprouting, synaptogenesis, and behavioral recovery after neocortical infarction in rats. Stroke 26:2135-2144.

Trachtenberg JT, Chen BE, Knott GW, Feng G, Sanes JR, Welker E, Svoboda $\mathrm{K}$ (2002) Long-term in vivo imaging of experience-dependent synaptic plasticity in adult cortex. Nature 26:788-794.

Ward NM, Sharkey J, Marston HM, Brown VJ (1998) Simple and choice reaction-time performance following occlusion of the anterior cerebral arteries in the rat. Exp Brain Res 123:269-281.

Whishaw IQ, Pellis SM, Gorny BP, Pellis VC (1991) The impairments in reaching and the movements in compensation in rats with motor cortex lesions: an endpoint, videorecording, and movement notation analysis. Behav Brain Res 42:77-91. 